\title{
ON ALMOST $C(\alpha)$-MANIFOLD SATISFYING SOME CONDITIONS ON THE WEYL PROJECTIVE CURVATURE TENSOR
}

\author{
ÜMİT YILDIRIM
}

\begin{abstract}
In the present paper, we have studied the curvature tensors of almost $C(\alpha)$-manifolds satisfying the conditions $P(\xi, X) R=0$, $P(\xi, X) \widetilde{Z}=0, P(\xi, X) P=0, P(\xi, X) S=0$ and $P(\xi, X) \widetilde{C}=0$. According these cases, we classified almost $C(\alpha)$-manifolds.
\end{abstract}

\section{INTRODUCTION}

In [10], authors studied the Weyl projective curvature tensor in an $N(k)$ contact metric manifold and classified $N(k)$-contact metric manifolds.

In [3] and [9], we searched the properties of curvature tensors of an almost $C(\alpha)$-manifold satisfying $\widetilde{Z}(\xi, X) R=\widetilde{Z}(\xi, X) \widetilde{Z}=\widetilde{Z}(\xi, X) S=\widetilde{Z}(\xi, X) P=0$ and Ricci semi-symmetric, projective semi-symmetric, quasi-conformal semisymmetric.

De U. C. and Sarkar A. [4] studied properties of projective curvature tensor to generalized Sasakian space form. Atçeken M. [2] studied generalized Sasakian space form satisfying certain conditions on the concircular curvature tensor. Özgür M. and De U. C. [6] researched some certain curvature conditions satisfied by quasi-conformal curvature tensor in Kenmotsu manifolds. Arslan K., Murathan C. and Özgür C. produced the works on contact manifold curvature tensor[1].

Motivated by the studies of the above authors, in this paper we classify almost $C(\alpha)$-manifolds, which satisfy the curvature conditions $P(\xi, X) R=0$, $P(\xi, X) \widetilde{Z}=0, P(\xi, X) P=0, P(\xi, X) S=0$ and $P(\xi, X) \widetilde{C}=0$, where $P$ is the Weyl projective curvature tensor, $\widetilde{Z}$ is the concircular curvature tensor, $S$ is the Ricci tensor and $\widetilde{C}$ is quasi-conformal curvature tensor.

2000 Mathematics Subject Classification. 53C15, 53C44, 53D10.

Key words and phrases. Almost $C(\alpha)$-manifold, weyl projective curvature tensor, concircular curvature tensor, real space form. 


\section{Preliminaries}

An odd-dimensional Riemannian manifold $(M, g)$ is said to be an almost co-Hermitian or almost contact metric manifold if there exist on $M$ a $(1,1)$ tensor field $\phi$, a vector field $\xi$ (called the structure vector field) and a 1-form $\eta$ such that

$$
\begin{gathered}
\eta(\xi)=1, \quad \phi^{2} X=-X+\eta(X) \xi \\
g(\phi X, \phi Y)=g(X, Y)-\eta(X) \eta(Y), \\
\phi \xi=0, \quad \eta \circ \phi=0,
\end{gathered}
$$

for any vector field $X, Y$ on $M$.

The Sasaki form (or fundamental 2-form) $\Phi$ of an almost co-Hermitian manifold $(M, g, \phi, \xi, \eta)$ is defined by

$$
\Phi(X, Y)=g(X, \phi Y)
$$

for all $X, Y$ on $\in \chi(M)$ and this form satisfies $\eta \wedge \Phi^{n} \neq 0$. This means that every almost co-Hermitian manifold is orientable and $(\eta, \Phi)$ defines an almost cosymplectic structure on $M$. If this associated structure is cosymplectic $(d \Phi=d \eta=0), M$ is called an almost co-Kähler manifold. The associated almost cosymplectic structure is a contact structure and is an almost Sasakian manifold when $\Phi=d \eta$. It is well known that every contact manifold has an almost Sasakian structure.

The Nijenhuis tensor of the (1,1)-tensor field $\phi$ is the $(1,2)$-tensor field $[\phi, \phi]$ defined by

$$
[\phi, \phi](X, Y)=\phi^{2}[X, Y]+[\phi X, \phi Y]-\phi[\phi X, Y]-\phi[X, \phi Y]
$$

where $[X, Y]$ is the Lie bracket of $X, Y \in \chi(M)$.

On the other hand, an almost co-complex structure is called integrable if $[\phi, \phi]=0$ and normal if $[\phi, \phi]+2 d \eta \otimes \xi=0$. A co-Kähler manifold (or normal cosymplectic manifold) is an integrable (or equivalently, a normal) almost contact Kähler manifold, while a Sasakian manifold is a normal almost Sasakian manifold $[5]$.

The Riemannian connections $\nabla$ of Sasakian, co-Kähler and Kenmotsu manifolds have some well known properties which allow us to characterize these manifolds. 
Theorem 2.1. Let $(M, g, \phi, \xi, \eta)$ be an almost co-Hermitian manifold with Riemannian connection $\nabla$. Then

(i) $\quad M$ is co-Kählerian if and only if $\nabla \phi=0$,

(ii) $\quad M$ is Sasakian if and only if

$$
\left(\nabla_{X} \phi\right) Y=g(X, Y) \xi-\eta(Y) X,
$$

(iii) $\quad M$ is Kenmotsu manifold if and only if

$$
\left(\nabla_{X} \phi\right) Y=g(\phi X, Y) \xi-\eta(Y) \phi X .
$$

for all $X, Y \in \chi(M)[5]$.

Theorem 2.2. $\xi$ is Killing vector field for co-Kähler and Sasaki manifolds, i.e.

$$
g\left(\nabla_{X} \xi, Y\right)+g\left(X, \nabla_{Y} \xi\right)=0
$$

while for Kenmotsu manifolds we have

$$
g\left(\nabla_{X} \xi, Y\right)-g\left(X, \nabla_{Y} \xi\right)=0 .
$$

for all $X, Y \in \chi(M)[5]$.

Theorem 2.3. Let $R$ be the Riemann curvature tensor on $M$. For all $X, Y, Z, W \in \chi(M)$, we have

(i) for $M$ co-Kählerian:

$$
R(X, Y, Z, W)=R(X, Y, \phi Z, \phi W)
$$

(ii) for $M$ Sasakian:

$$
\begin{aligned}
R(X, Y, Z, W) & =R(X, Y, \phi Z, \phi W)-g(X, Z) g(Y, W)+g(X, W) g(Y, Z) \\
& +g(X, \phi Z) g(Y, \phi W)-g(X, \phi W) g(Y, \phi Z) ;
\end{aligned}
$$

(iii) for a Kenmotsu manifold $M$ :

$$
\begin{aligned}
R(X, Y, Z, W) & =R(X, Y, \phi Z, \phi W)+g(X, Z) g(Y, W)-g(X, W) g(Y, Z) \\
& -g(X, \phi Z) g(Y, \phi W)+g(X, \phi W) g(Y, \phi Z),
\end{aligned}
$$

Definition 2.4. An almost $C(\alpha)$-manifold $M$ is an almost co-Hermitian manifold such that the Riemann curvature tensor satisfies the following property: $\exists \alpha \in R$ such that

$$
\begin{aligned}
R(X, Y, Z, W) & =R(X, Y, \phi Z, \phi W)+\alpha\{-g(X, Z) g(Y, W)+g(X, W) g(Y, Z) \\
(2.5) & +g(X, \phi Z) g(Y, \phi W)-g(X, \phi W) g(Y, \phi Z)\} .
\end{aligned}
$$

for all $X, Y, Z, W \in \chi(M)$. 
Moreover, if such a manifold has constant $\phi$-sectional curvature equal to $c$, then its curvature tensor is given by

$$
\begin{aligned}
R(X, Y) Z & =\left(\frac{c+3 \alpha}{4}\right)\{g(Y, Z) X-g(X, Z) Y\} \\
& +\left(\frac{c-\alpha}{4}\right)\{g(X, \phi Z) \phi Y-g(Y, \phi Z) \phi X+2 g(X, \phi Y) \phi Z\} \\
& +\left(\frac{c-\alpha}{4}\right)\{\eta(X) \eta(Z) Y-\eta(Y) \eta(Z) X+g(X, Z) \eta(Y) \xi \\
& -g(Y, Z) \eta(X) \xi\} .
\end{aligned}
$$

A normal almost $C(\alpha)$-manifold is called $C(\alpha)$-manifold[5].

Co-Kählerian, Sasakian and Kenmotsu manifolds are, respectively, $C(0)$, $C(1)$ and $C(-1)$-manifolds.

Theorem 2.5. An almost co-Hermitian manifold $M$ is $\alpha$-Sasakian if and only if for all $X, Y \in \chi(M)$

$$
\left(\nabla_{X} \phi\right) Y=\alpha\{g(X, Y) \xi-\eta(X) Y\}
$$

(ii) If $M$ is $\alpha$-Sasakian, then $\xi$ is a Killing vector field and

$$
\nabla_{X} \xi=-\alpha \phi X
$$

for all $X \in \chi(M)$.

(iii) An $\alpha$-Sasakian manifold is a $C\left(\alpha^{2}\right)$-manifold[5].

Theorem 2.6. An almost co-Hermitian manifold is an $\alpha$-Kenmotsu manifold if and only if

$$
\begin{gathered}
\left(\nabla_{X} \phi\right) Y=\alpha\{g(\phi X, Y) \xi-\eta(Y) \phi X\}, \\
\nabla_{X} \xi=\alpha\{-X+\eta(X) \xi\}
\end{gathered}
$$

for all $X, Y \in \chi(M)$.

(ii) An $\alpha$-Kenmotsu manifold is a $C\left(-\alpha^{2}\right)$-manifold[5].

The concept of quasi-conformal curvature tensor was defined by K. Yano and S. Sawaki [8]. Quasi-conformal curvature tensor of a $(2 n+1)$-dimensional Riemannian manifold is defined as

$$
\begin{aligned}
\widetilde{C}(X, Y) Z & =a R(X, Y) Z+b[S(Y, Z) X-S(X, Z) Y+g(Y, Z) Q X \\
& -g(X, Z) Q Y]-\frac{r}{2 n+1}\left[\frac{a}{2 n}+2 b\right][g(Y, Z) X-g(X, Z) Y]
\end{aligned}
$$


where, $a$ and $b$ are arbitrary constants, $Q, S$ and $r$ denote the Ricci operator, Ricci tensor and scalar curvature of manifold, respectively. If $\widetilde{C}=0$, then manifold is said to be quasi-conformal flat.

Let $M$ be $(2 n+1)$-dimensional Riemannian manifold. The Weyl projective curvature tensor field is defined by $[7]$

$$
P(X, Y) Z=R(X, Y) Z-\frac{1}{2 n}[S(Y, Z) X-S(X, Z) Y]
$$

for any $X, Y, Z \in \chi(M)$.

Let $(M, g)$ be an $(2 n+1)$-dimensional Riemannian manifold. Then the concircular curvature tensor $\widetilde{Z}$ is defined by

$$
\widetilde{Z}(X, Y) Z=R(X, Y) Z-\frac{r}{2 n(2 n+1)}(g(Y, Z) X-g(X, Z) Y),
$$

for all $X, Y, Z \in \chi(M)$, where $r$ is the scalar curvature of $M[7]$.

\section{An almost $C(\alpha)$-Manifold Satisfying Certain Conditions on the Weyl Projective Curvature Tensor}

In this section, we will give the main results for this paper.

Let $M$ be $(2 n+1)$-dimensional almost $C(\alpha)$-manifold and we denote the Riemannian curvature tensor of $R$, then we have from (2.6), for $X=\xi$,

$$
R(\xi, Y) Z=\alpha\{g(Y, Z) \xi-\eta(Z) Y\} .
$$

In the same way, choosing $Z=\xi$ in (2.6), we have

$$
R(X, Y) \xi=\alpha\{\eta(Y) X-\eta(X) Y\} .
$$

In (3.2), choosing $Y=\xi$, we obtain

$$
R(X, \xi) \xi=\alpha\{X-\eta(X) \xi\} .
$$

Also, from (2.6), we obtain

$$
\eta(R(X, Y) Z)=\alpha\{g(Y, Z) \eta(X)-g(X, Z) \eta(Y)\} .
$$

In the same way choosing $X=\xi$ in (2.11), we have

$$
\begin{aligned}
\widetilde{C}(\xi, Y) Z & =\left\{a \alpha+2 n \alpha b-\frac{r}{2 n+1}\left[\frac{a}{2 n}+2 b\right]\right\}\{g(Y, Z) \xi-\eta(Z) Y\} \\
& +b\{S(Y, Z) \xi-\eta(Y) Q Y\} .
\end{aligned}
$$


In (3.5), choosing $Z=\xi$, we obtain

$$
\begin{aligned}
\widetilde{C}(\xi, Y) \xi & =\left\{a \alpha+2 n \alpha b-\frac{r}{2 n+1}\left[\frac{a}{2 n}+2 b\right]\right\}\{\eta(Y) \xi-Y\} \\
& +b\{2 n \alpha \eta(Y) \xi-Q Y\}
\end{aligned}
$$

Also, from (2.13) we have

$$
\widetilde{Z}(\xi, X) Y=\left\{\alpha-\frac{r}{2 n(2 n+1)}\right\}\{g(X, Y) \xi-\eta(Y) X\}
$$

and

$$
\widetilde{Z}(\xi, X) \xi=\left\{\alpha-\frac{r}{2 n(2 n+1)}\right\}\{\eta(X) \xi-X\} .
$$

Also, from (2.12), we have

$$
P(\xi, Y) Z=\alpha g(Y, Z) \xi-\frac{1}{2 n} S(Y, Z) \xi
$$

From (2.6), we can state

$$
\begin{aligned}
R\left(X, e_{i}\right) e_{i}+R\left(X, \phi e_{i}\right) \phi e_{i}+R(X, \xi) \xi & =\sum_{i=1}^{n}\left\{( \frac { 3 \alpha + c } { 4 } ) \left\{n X-g\left(X, e_{i}\right) e_{i}+n X\right.\right. \\
& \left.-g\left(X, \phi e_{i}\right) \phi e_{i}+X-g(X, \xi) \xi\right\} \\
& +\left(\frac{c-\alpha}{4}\right)\left\{3 g\left(X, \phi e_{i}\right) \phi e_{i}-2 n \eta(X) \xi\right. \\
& \left.\left.+3 g\left(X, \phi^{2} e_{i}\right) \phi^{2} e_{i} \eta(X) \xi-X\right\}\right\},
\end{aligned}
$$

for $\left\{e_{1}, e_{2}, \ldots, e_{n}, \phi e_{1}, \ldots, \phi e_{n}, \xi\right\}$ orthonormal basis of $M$. From (3.10), for $Y \in \chi(M)$, we obtain

$$
\begin{aligned}
S(X, Y) & =\left(\frac{\alpha(3 n-1)+c(n+1)}{2}\right) g(X, Y) \\
& +\left(\frac{(\alpha-c)(n+1)}{2}\right) \eta(X) \eta(Y),
\end{aligned}
$$

which is equivalent to

$$
Q X=\left(\frac{\alpha(3 n-1)+c(n+1)}{2}\right) X+\left(\frac{(\alpha-c)(n+1)}{2}\right) \eta(X) \xi
$$

From (3.11), we can give the following corollary.

Also, from (3.11), we can easily see

$$
\begin{gathered}
r=n[\alpha(3 n+1)+c(n+1)], \\
S(X, \xi)=2 n \alpha \eta(X),
\end{gathered}
$$


and

$$
Q \xi=2 n \alpha \xi
$$

Theorem 3.1. Let $M$ be (2n+1)-dimensional an almost $C(\alpha)$-manifold.Then, $P(\xi, X) R=0$ if and only if $M$ reduce real space form with constant sectional curvature $c$.

Proof. Suppose that $P(\xi, X) R=0$. Then, we have

$$
\begin{aligned}
(P(\xi, X) R)(U, W) Z & =P(\xi, X) R(U, W) Z-R(P(\xi, X) U, W) Z \\
& -R(U, P(\xi, X) W) Z-R(U, W) P(\xi, X) Z \\
& =0 .
\end{aligned}
$$

Using (3.9) in (3.16), we obtain

$$
\begin{array}{ll}
= & \alpha\{g(X, R(U, W) Z) \xi-g(X, U) R(\xi, W) Z \\
- & g(X, W) R(U, \xi) Z-g(X, Z) R(U, W) \xi\} \\
- & \frac{1}{2 n}\{S(X, R(U, W) Z) \xi-S(X, U) R(\xi, W) Z \\
- & S(X, W) R(U, \xi) Z-S(X, Z) R(U, W) \xi\} \\
= & 0 .
\end{array}
$$

Putting $U=\xi$ in (3.17) and using the equations (3.1) and (3.2), we have

$$
\begin{aligned}
\frac{1}{2 n} S(X, W) \eta(Z) & =\alpha\{g(X, W) \eta(Z)+\eta(Z) \eta(X) \eta(W) \\
& -g(W, Z) \eta(X)\}
\end{aligned}
$$

which implies that

$$
S(X, W)=2 n \alpha g(X, W) .
$$

So, the almost $C(\alpha)$-manifold is an Einstein manifold. In this case $r=$ $2 n \alpha(2 n+1)$. Taking into account of $(3.13)$, we obtain $\alpha=c$, which implies that

$$
R(X, Y) Z=c\{g(Y, Z) X-g(X, Z) Y\} .
$$

The converse is obvious.

Theorem 3.2. Let $M$ be $(2 n+1)$-dimensional an almost $C(\alpha)$-manifold. Then, $P(\xi, X) \widetilde{Z}=0$ if and only if $M$ is a real space form with sectional curvature $c$.

Proof. Suppose that $P(\xi, X) \widetilde{Z}=0$, we have

$$
\begin{aligned}
(P(\xi, X) \widetilde{Z})(U, W) Z & =P(\xi, X) \widetilde{Z}(U, W) Z-\widetilde{Z}(P(\xi, X) U, W) Z \\
& -\widetilde{Z}(U, P(\xi, X) W) Z-\widetilde{Z}(U, W) P(\xi, X) Z \\
& =0 .
\end{aligned}
$$


Using (2.13) and (3.9) in (3.19), we obtain

$$
\begin{aligned}
0 & =\alpha\{g(X, \widetilde{Z}(U, W) Z) \xi-g(X, U) \widetilde{Z}(\xi, W) Z-g(X, W) \widetilde{Z}(U, \xi) Z \\
& -g(X, Z) \widetilde{Z}(U, W) \xi\}-\frac{1}{2 n}\{S(X, \widetilde{Z}(U, W) Z) \xi-S(X, U) \widetilde{Z}(\xi, W) Z \\
(3.20)- & S(X, W) \widetilde{Z}(U, \xi) Z-S(X, Z) \widetilde{Z}(U, W) \xi\} .
\end{aligned}
$$

In (3.20), choosing $U=\xi$ and using (2.13), (3.7), (3.8) and (3.14), we have

$$
\begin{aligned}
0 & =\left[\alpha-\frac{r}{2 n(2 n+1)}\right]\{\alpha g(X, Z) W-\alpha g(X, Z) \eta(W) \xi \\
& -\alpha g(X, W) \eta(Z) \xi+\frac{1}{2 n} S(X, W) \eta(Z) \xi+\frac{1}{2 n} S(X, Z) \eta(W) \xi \\
& \left.-\frac{1}{2 n} S(X, Z) W\right\} .
\end{aligned}
$$

Inner product both sides of the equation by $\xi$, we have

$$
\left[\alpha-\frac{r}{2 n(2 n+1)}\right]\left\{\frac{1}{2 n} S(X, W)-\alpha g(X, W)\right\}=0
$$

If $r=2 n \alpha(2 n+1)$, from (3.13), we obtain $\alpha=c$. This implies that $M$ is a real space form. Otherwise $S(X, Y)=2 n \alpha g(X, Y)$. This tells us $r=2 n \alpha(2 n+1)$.

Theorem 3.3. Let $M$ be $(2 n+1)$-dimensional an almost $C(\alpha)$-manifold.Then, $P(\xi, Y) P=0$ if and only if $M$ reduce real space form with constant sectional curvature $c=\alpha$.

Proof. Suppose that $P(\xi, Y) P=0$, we have

$$
\begin{aligned}
(P(\xi, Y) P)(Z, U) W & =P(\xi, Y) P(Z, U) W-P(P(\xi, Y), U) W \\
& -P(Z, P(\xi, Y) U) W-P(Z, U) P(\xi, Y) W \\
& =0 .
\end{aligned}
$$

Using (3.9) in (3.22), we have

$$
\begin{aligned}
0 & =\alpha\left\{g(Y, P(Z, U) W) \xi-\alpha g(Y, Z) g(U, W) \xi+\frac{1}{2 n} g(Y, Z) S(U, W) \xi\right. \\
& \left.-\frac{1}{2 n} g(Y, U) S(Z, W) \xi+\alpha g(Y, U) g(W, Z) \xi\right\} \\
& +\frac{1}{2 n}\left\{-S(Y, P(Z, U) W) \xi+\alpha g(U, W) S(Y, Z) \xi-\frac{1}{2 n} S(Y, Z) S(U, W) \xi\right. \\
(3.23)+ & \left.\frac{1}{2 n} S(Y, U) S(Z, W) \xi-\alpha S(Y, U) g(W, Z) \xi\right\} .
\end{aligned}
$$

Using the equations (2.12) and (3.11) in (3.23), we obtain

$$
\left[\frac{(\alpha-c)(n+1)}{4 n}\right][R(Z, U) W-\alpha\{g(U, W) Z-g(W, Z) U\}]=0,
$$

which proves our assertion. 
Theorem 3.4. Let $M$ be (2n+1)-dimensional an almost $C(\alpha)$-manifold.Then, $P(\xi, Y) \widetilde{C}=0$ if and only if $M$ has either $\alpha$-sectional curvature or it is an Einstein manifold.

Proof. Suppose that $P(\xi, Y) \widetilde{C}=0$, we have

$$
\begin{aligned}
(P(\xi, Y) \widetilde{C})(Z, U) W & =P(\xi, Y) \widetilde{C}(Z, U) W-\widetilde{C}(P(\xi, Y) Z, U) W \\
& -\widetilde{C}(Z, P(\xi, Y) U) W-\widetilde{C}(Z, U) P(\xi, Y) W \\
& =0 .
\end{aligned}
$$

Using (3.9) in (3.24), we obtain

$$
\begin{aligned}
0 & =\alpha\{g(Y, \widetilde{C}(Z, U) W) \xi-g(Y, Z) \widetilde{C}(\xi, U) W \\
& -g(Y, U) \widetilde{C}(Z, \xi) W-g(Y, W) \widetilde{C}(Z, U) \xi\} \\
& -\frac{1}{2 n}\{S(Y, \widetilde{C}(Z, U) W) \xi-S(Y, Z) \widetilde{C}(\xi, U) W \\
& -S(Y, U) \widetilde{C}(Z, \xi) W-S(Y, W) \widetilde{C}(Z, U) \xi\} \\
& =0 .
\end{aligned}
$$

In (3.25), choosing $Z=\xi$ and using (3.5) and (3.6), we obtain

$$
\begin{aligned}
0 & =\alpha\left\{a \alpha+2 n \alpha b-\frac{r}{2 n+1}\left[\frac{a}{2 n}+2 b\right]\right\}\{g(Y, Q U)-2 n \alpha g(Y, U)\} \\
(3.26) & +b\{S(Y, Q U)-S(U, Y)\}
\end{aligned}
$$

Using (3.12) in (3.26) and choosing $U=\phi U$, we have

$\left[\frac{(n+1)(c-\alpha)}{2}\right]\left\{b S(\phi U, Y)+\left[a \alpha+2 n \alpha b-\frac{r}{2 n+1}\left[\frac{a}{2 n}+2 b\right]\right] g(\phi U, Y)\right\}=0$.

The proof is completed.

Theorem 3.5. Let $M$ be $(2 n+1)$-dimensional an almost $C(\alpha)$-manifold. Then, $P(\xi, X) S=0$ if and only if $M$ is an Einstein manifold.

Proof. Suppose that $P(\xi, X) S=0$, we have

$$
S(P(\xi, X) U, W)+S(U, P(\xi, X), W)=0 .
$$

In (3.27), using (3.9), we have

$$
\alpha\{g(X, W) \xi+g(X, U) \xi\}-\frac{1}{2 n}\{S(X, W) \xi+S(X, U) \xi\}=0
$$

Inner product both sides of (3.28) by $\xi \in \chi(M)$, and choosing $U=\xi$, we have

$$
S(X, W)=2 n \alpha g(X, W)
$$

So, $M$ is an Einstein manifold. 


\section{REFERENCES}

[1] Arslan K., Murathan C. and Özgür C., On contact manifolds satisfying certain curvature conditions, An. Univ. Bucuresti Math. (2000), 49(2), 17-26.

[2] Atçeken M., On generalized Sasakian space forms satisfying certain conditions on the concircular curvature tensor, Bulletin of Mathematical Analysis and Applications, $6(1)(2014), 1-8$.

[3] Atçeken M. and Yıldırım Ü., On almost $C(\alpha)$-manifold satisfying certain conditions on the concircular curvature tensor, Pure and Applied Mathematics Journal, Special Issue: Applications of Geometry. Vol. 4, No. 1-2, (2015), pp. 31-34. doi: 10.11648/j.pamj.s.2015040102.18.

[4] De U.C. and Sarkar A., On the Projective curvature tensor of Generalized Sasakianspace forms, Quaestiones Mathematicae, 33(2010), 245-252.

[5] Janssens D. and Vanhecke L., Almost contact structure and curvature tensors, Kodai Math. J., 4(1981), 1-27.

[6] Özgür C. and De U.C., On the quasi-conformal curvature tensor of a Kenmotsu manifold, Mathematica Pannonica, (2006), 17(2), 221-228.

[7] Yano K. and Kon M., Structures manifolds, Singapore, World Scientific, (1984).

[8] Yano K. and Sawaki S., Riemannian manifolds admitting a conformal transformation group, J. Diff. Geom. 2(1968), 161-184.

[9] Yıldırım Ü. and Atçeken M., On curvature tensors of an almost $C(\alpha)$-manifold, International Journal of Physical and Mathematical Sciences, Vol. 5, No. 1(2015), 53-61.

[10] Yıldız A., De U. C., Murathan C. and Arslan K., On the weyl projective curvature tensor of an $N(k)$-contact metric manifold, Mathematica Pannonica, 21/1 (2010), 1-14.

Gaziosmanpasa University, Faculty of Arts and Sciences, Department of MathEMATICS, 60100 TOKAT/TURKEY

E-mail address: umit.yildirim@gop.edu.tr 